Pierric Giraud

Emmanuel Jouanneau

Françoise Borson-Chazot

Michel Lanteri-Minet

Guy Chazot

\section{Cluster-like headache: literature review}

Key words Cluster-like headaches • Symptomatic cluster headache • Tumor - Cluster headache physiopathology
Neurology Service A,

Hôpital de la Région Annecienne,

1 Avenue du Tresum, 74000 Annecy, France

e-mail: glap@wanadoo.fr

Tel.: +33-450883337

Fax: $+33-450883052$

\section{E. Jouanneau}

Neurosurgery Service D,

Neurological Hospital,

Lyon, France

F. Borson-Chazot

Nuclear Medicine Service,

Neurological Hospital,

Lyon, France

M. Lanteri-Minet

Department for the Treatment and

Evaluation of Pain,

Pasteur Hospital,

Nice, France

\section{G. Chazot}

Neurology Service D

Neurological Hospital

Lyon, France

\begin{abstract}
Cluster headache $(\mathrm{CH})$ is the most frequent primary trigeminal autonomic cephalalgia

(Goadsby, 2001). Clinical symptoms have been clearly identified and are described in the IHS classification. Cluster-like headache (CLH), or symptomatic cluster headache, is a rare syndrome in which $\mathrm{CH}$ is associated with or disclosed by another neurological disease. The relationship between the two diseases is debated on a spatial and temporal bases. Relief of $\mathrm{CH}$ symptoms on treatment of the associated disease is a further argument. However, the simultaneous occurrence of the two diseases may be coincidental. First, the characteristics and pathogenesis of $\mathrm{CH}$ are reviewed. Second, published examples of CLH are described, including the pathophysiology proposed by the authors in each particular situation. In the final part, criteria for the identification of CLH in clinical practice are developed.
\end{abstract}

\section{Introduction}

Cluster headache $(\mathrm{CH})$ is the most frequent primary trigeminal autonomic cephalalgia [1]. Characteristics of headache, type and location of pain, autonomic symptoms and duration of attacks, as well as the cluster period, are described in the International Headache Society classification [2]. The precise description of symptoms contrasts with the limited knowledge of $\mathrm{CH}$ pathogenesis and pathophysiology. In fact, $\mathrm{CH}$ remains an original chronobiological disease where the combined action of several different neurological structures produces the pathology. Among the hypotheses, activation of the trigeminovascular system (TVS) seems to be of primary importance during attacks at cluster period [3]. The role of the hypothalamus and brainstem in controlling the activity of the spinal trigeminal nucleus is also wellestablished [4].

In the literature, few observations have been reported as cluster-like headache (CLH) or symptomatic cluster headache. In these cases, $\mathrm{CH}$ is associated with another neurological disease, most often a pituitary adenoma, vascular 
loops, fungal sinusitis or even a tumor. The relationship between the two diseases is frequently debated. The main arguments supporting their relationship are the spatial location and a temporal link. Indeed, diseases described in association with $\mathrm{CH}$ are usually located close to anatomical structures potentially involved in $\mathrm{CH}$. Moreover, the delay between their occurrence or detection and $\mathrm{CH}$ is usually not far-spaced, making a temporal link possible. As a consequence, the suspicion of their implication in CLH is reinforced.

In the first section of this paper, characteristics and pathogenesis of $\mathrm{CH}$ are reviewed. In the second part, cases of cluster-like headache published from 1980 to 2001 are described. In the final part, criteria helpful for the identification of CLH are developed, their presence requiring subsequent complementary investigations.

\section{Cluster headache}

Cluster headache $(\mathrm{CH})$ is a rare facial disease with a prevalence of $0.1 \%$ in the general population. $\mathrm{CH}$ is characterized by unilateral paroxysmal pain mainly located in the orbital region with a duration of 15-180 minutes and is constantly associated, during attacks, with homolateral autonomic symptoms of the face (Table 1). Evolution is either chronic or episodic. The chronic form is diagnosed when disease duration exceeds one year or when the remission period lasts less than 14 days. During the active phase of the disease (cluster periods), attacks are mainly nocturnal, repetitive and have a daily periodicity. Attacks may be precipitated by alcohol ingestion, histamine or nitroglycerin. Male preponderance, as well as some lifestyle habits, such as alcohol intake and cigarette smok- ing, head injury or even stressful work conditions are also strongly associated with this disease [3-5]. When all symptoms are present, complementary radiological or electrophysiological exams are considered unnecessary. Treatment relies on symptomatic and prophylactic drugs. Symptomatic treatment acts on pain and autonomic symptoms during attacks. Sumatriptan, inhalation of oxygen, or even local anesthetics within the foramen sphenopalatinum are the most common. Prophylactic therapies are used to delay attacks and to limit the necessity for acute treatment. Methysergide, verapamil, corticosteroid and lithium are classic treatments.

In $\mathrm{CH}$, the mechanism of pain and development of autonomic symptoms remains debatable. However, the implication of the trigeminovascular system (TVS) as well as a transitory dysregulation of autonomic fibers under hypothalamic control are the most accepted theories.

The TVS is abnormally stimulated as demonstrated by the increase of calcitonin gene related peptide (CGRP) or vasoactive intestinal peptide (VIP) in plasma from the jugular vein, expression of c-fos in the spinal trigeminal nucleus and the effect of triptan during attacks $[6,7]$. This pathologic activation could be due either to recurrent transitory inflammation in the cavernous sinus, according to Moskowitz [7] and Hardebo [8, 9], or to abnormal discharge in the bipolar neurons of Gasser's ganglion [8, 9]. In the first theory, modification of the size of the intracavernous vessels could cause lesions in the sympathetic and nociceptive fibers situated in the wall of the intracranial internal carotid $[3,8,10]$. The modification of the orbital venous blood flow observed in $\mathrm{CH}$, as well as the possibility of provoking $\mathrm{CH}$ by powerful vasodilators, support the vascular theory of $\mathrm{CH}$ produced by a local factor located in the cavernous sinus [7, 11]. Moreover, the fixed site of pain, as well as the ipsilateral autonomic symptoms, are in

Table 1 Diagnostic criteria for cluster headache [2]

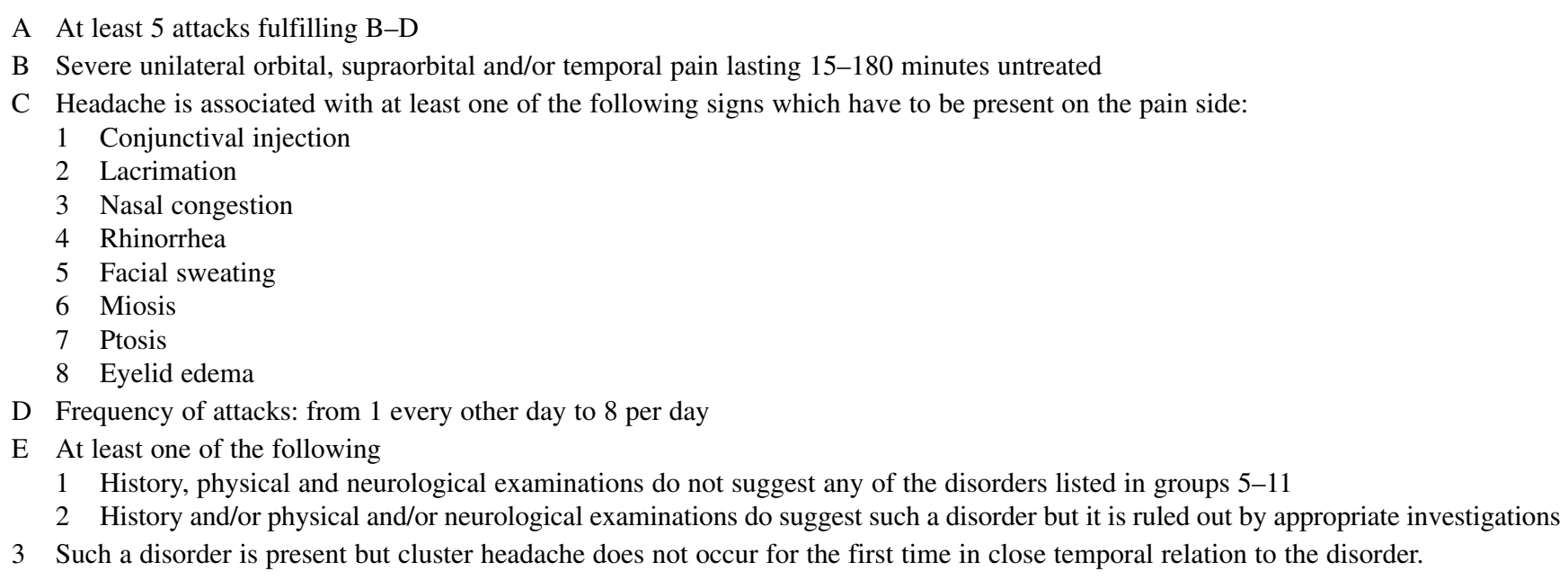


accordance with such a theory [11]. Nevertheless, a purely vasogenic theory fails to explain several other aspects of the disease, such as the cluster period, or even the disruption of several biological rhythms, or the spontaneous arrest of attacks. For these reasons, the hypothalamus and central structures have been implicated. Hardebo [9] and Sicuteri [10] hypothesized the involvement of trigeminal substance $\mathrm{P}$ neurons in $\mathrm{CH}$. Activation of substance $\mathrm{P}$ neurons in ophthalmic and maxillary nerves provokes intense pain and autonomic symptoms by stimulation of mast cells and liberation of inflammatory mediators. The pathophysiology of $\mathrm{P}$ neurons remains unknown. Among the theories, a reduction in descending inhibitory control of the spinal trigeminal nucleus under the influence of hypothalamic-limbic structures is hypothesized [12]. This theory explains the pain, the autonomic signs and also the chronobiological aspects of the disease. Other theories have been previously suggested but should be discussed in relation to lesion sites.

\section{Cluster-like headache}

\section{Definition}

The definition of CLH is not given by the IHS. This is probably the cause of different interpretations of this term in literature. In fact, in published cases of CLH, there is a wide variety of heterogeneous observations explained by diverse definitions and classifications.

For some authors, the IHS criteria are fundamental. Thus, CLH only includes cases that fully adhere to the IHS description of $\mathrm{CH}$ differing only by the presence of another disease which may be involved. Greve and Mai [13] or Porta-Etessam et al. [14] described such observations in which the occurrence of another neurological disease eliminates the diagnosis of $\mathrm{CH}$ even if all the symptoms adhere to IHS criteria. In these cases, several questions remain unanswered. For example, the delay between the two diseases, which would suggest an obvious link, is unknown. Moreover, treatment of the second disease should cure CLH but there is no consensus on the delay

Table 2 Diagnostic criteria for cluster-like headache

IHS criteria for cluster headache are met

Physical examination during and outside attacks is normal

A lesion is located in a site where TVS can be activated

Headache is cured after treatment of the second disease without recurrence

IHS, International Headache Society; TVS, trigeminovascular system required to consider that $\mathrm{CH}$ has definitively disappeared. Additionally, the second disease should necessarily be situated close to the trigemino vascular system, otherwise, it should not be implicated. Because of these difficulties, other authors insisted mostly on the type of pain as the main factor defining CLH. Therefore, the type and topography of pain, always unilateral, intense facial pain of short duration, justify the term CLH. Autonomic symptoms and perhaps an abnormal clinical examination outside attacks are sometimes noted but appear less important [15-18].

In this review, CLH cases were selected on the basis of four associated points (Table 2). First, headache should fulfill the IHS criteria for episodic or chronic $\mathrm{CH}$, both autonomic symptoms during attacks (Table 1) and duration of attacks. These factors are particularly important for case homogeneity. Second, physical and neurological examinations should be strictly normal during and after attacks, otherwise radiological investigations are required. This point should be included by authors in their observations. Third, the second neurological disease should be located close to the anatomical structure implicated in the pathogenesis of $\mathrm{CH}$. The last important factor should be the relief of CLH symptoms without recurrence after adequate treatment of the other neurological disease. However, we chose to overlook the delay between CLH and the discovery of a second neurological disease or the contrary. Moreover, the delay between treatment and relief of CLH symptoms was not necessarily specified. In this way, the relationship between the two diseases was not limited by temporal aspects.

\section{CLH and pathology}

A Medline research was conducted to identify all observations published as CLH, or symptomatic cluster headache, and $\mathrm{CH}$ associated with another disease between 1980 and 2001. The difficulty was in detecting all observations as some are described in $\mathrm{CH}$ series. Using this procedure, 68 cases were identified but some may have been overlooked. Cases were classed in relation to the site of the second neurological disease, that is, either in the face, in the cavernous sinus or its vicinity, in the cranial vessels or in various other brain areas (Table 3 ).

Before describing these observations, it should be pointed out that due to the small number of cases a coincidental event cannot be excluded nor can a direct relationship between the second neurological disease and the occurrence of CLH. The most thoroughly detailed observations were selected for each etiology to give an overall view of CLH. 
Table 3 Lesions associated with cluster-like headache as reported in the literature, classed by site

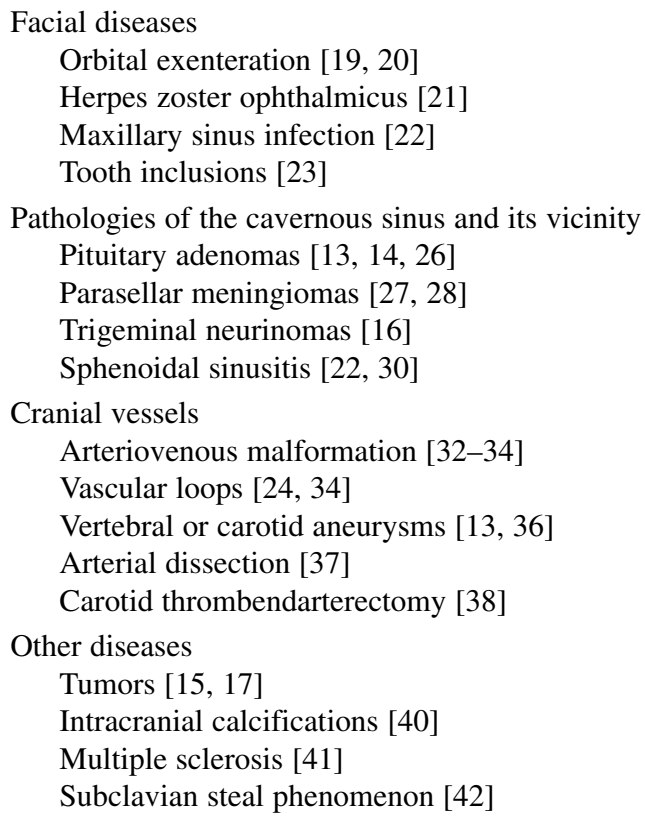

\section{CLH and facial diseases}

Associated facial diseases are mainly eye lesions but can also concern facial sinuses and teeth. Orbital exenteration was reported in 6 cases [19], one case being due to cancer and the others to traumatic lesions of the eye. In all observations, the disease appeared in men aged 21-56 years. CLH symptoms occurred within 3 weeks to 15 years of orbital extraction $[19,20]$. CLH is always ipsilateral to the eye lesion and prophylactic or symptomatic treatment is that of $\mathrm{CH}$. Damage is thought to provoke irritation of the orbital tissue causing pathologic stimulation of sensitive and autonomic fibers. Another example involving the ocular region in cases of $\mathrm{CH}$ is that induced by cutaneous viral infection. Sacquegna et al. [21] reported cases of CLH three months after herpes zoster ophthalmicus on the same side as previous viral infection. CLH proved to be particularly refractory to drugs. However, the rarity of this etiology suggests that it may be coincidental rather than due to a direct link between $\mathrm{CH}$ and viral infection. Maxillary sinusitis can also be associated with CLH. Takeshima et al. [22] described CLH revealing a maxillary sinus infection without any of the usual disorders associated with this disease. Rapidly, the persistence of pain outside attacks and the diminished frequency of attacks suggested that there was a second disease. To explain the relationship between the two diseases, the authors suggested that the trigeminal and autonomic fibers had been injured by infection. Tooth inclusions are rarely reported as causes of CLH. However, an impacted superior wisdom tooth was described as such by Romoli and Cudia [23]. The particularity of this observation is that dental extraction led to complete relief of CLH symptoms that had been present for 15 years. The tooth was within the pterygopalatine fossa, suggesting abnormal pressure or even lesion of the nervus intermedius fibers, the fifth nerve and the sympathetic postganglionic fibers of the superior cervical ganglion. This hypothesis supports the implication of nervus intermedius in $\mathrm{CH}$ [24].

CLH and pathologies of the cavernous sinus and its vicinity

This location remains one of the most frequent sites where lesions are detected and published for CLH.

Intrasellar tumors are mainly pituitary adenomas but few observations fulfill IHS criteria for $\mathrm{CH}$. In fact, in most cases, duration of pain is actually more prolonged than in $\mathrm{CH}$ and autonomic signs are frequently lacking [25]. Nevertheless, Tfelt-Hensen et al. [26], Greve and Mai [13] and Porta-Etassam et al. [14] described several, well-documented cases. In these observations, pituitary prolactinomas or growth-hormone producing adenomas were always macroadenomas with suprasellar and laterosellar infiltrations. Clinically, CLH is systematically located on the same side as cavernous sinus invasion. The duration of CLH was 31 years in one case whereas it was less than 2 years for the others. Partial efficacy of symptomatic and prophylactic treatments explained the complementary radiological examinations disclosing the macroadenoma. In the vicinity of pituitary gland, Hannertz [27] described a parasellar meningioma developing in the left lateral sinus narrowing the cavernous sinus veins. CLH was firstly episodic, then chronic and later transformed into painful homolateral ophthalmoplegia. For 37 years, symptoms were strictly compatible with $\mathrm{CH}$, confirmed by three independent clinicians. Nevertheless, retrospectively, Hannertz [27] postulated that, for him, CH was atypical. In fact, among signs that should have attracted attention, the author noticed that, for five years, the pain was moderately severe, there was no strict periodicity and prophylactic drugs had little effect. In spite of this, diagnosis was only made when the patient presented neurological signs on the same side as $\mathrm{CH}$. In this case, as in the previous ones, treatment of the meningioma was associated with definitive relief of CLH symptoms prompting Hannertz to suspect a direct link between the two diseases [27]. Lefevre et al. [28] reported another example of meningiomas discovered by CLH. Trigeminal neurinoma with large extension of the disease into the left cavernous sinus is another etiology for a laterosellar pathology [16]. CLH was typical and systematically located on the same side as main tumor development. One of the interesting points is the 
extension of the lesion to involve the cisternal part of the trigeminal nerve, the trigeminal ganglion and also contact with the intracavernous portion of the internal carotid. Observations of pituitary adenomas, parasellar meningiomas or neurinomas support the hypothesis that pain and autonomic symptoms in $\mathrm{CH}$ originate within the cavernous sinus region $[3,7,10]$. For some authors, one of the clues for a causal relationship between the two diseases lies in the relief of CLH symptoms without recurrence after treatment of the lesion [13, 14, 26]. Among arguments to explain such a relationship, Hannertz clearly demonstrated by orbital phlebography that venous drainage in the cavernous sinus was impaired by the tumor. All these diseases argument in favor of the role of the superior pericarotid cavernous sinus plexus. Several questions remain debatable, in particular, why does pain not persist if compression is the main mechanism of CLH in this situation? Moreover, for pituitary adenoma, Hall et al. [29] found silent pituitary adenomas in $10 \%$ of a random adult population using magnetic resonance imaging (MRI), while occult pituitary adenomas were described in 3\%-27\% of subjects at autopsy [30]. Therefore, the possibility of a rare coincidental association remains possible.

Infection of the sphenoidal sinus has also been exceptionally reported as a cause of CLH. As for pituitary adenomas, most of these observations do not strictly adhere to IHS criteria because they are associated with persistent neurologic symptoms between attacks or a lower frequency of attacks [22]. In contrast, Zanchin et al. [31] described a woman who suffered from fungal sphenoidal sinusitis, first associated with typical CLH but without the usual symptoms associated with sinus disease. The sinusitis due to an aspergilloma was treated with surgery and daily itraconazole. The relief of CLH symptoms suggested a link between the diseases. The authors hypothesized that CLH symptoms were due to permanent stimulation of the sensory fibers originating from the posterior ethmoidal nerve. Autonomic fibers depending on the sphenopalatine ganglion were also injured by inflammatory cells.

\section{CLH and cranial vessels}

Arteriovenous malformation (AVM), vascular loops, vertebral or carotid aneurysms, arterial dissection or carotid thrombendarterectomy have been reported in cases of CLH. They represented, with pituitary adenoma, the main example of diseases discovered in symptomatic $\mathrm{CH}$. However, in cases of AVM, most observations were not actually CLH as previously defined as the pain was not typical and autonomic symptoms were constantly lacking $[32,33]$. On the contrary, Gawel et al. [34] reported a case of CLH localized on the same side as the artery implicated in AVM. When right anterior cerebral artery supplied the AVM, CLH was on the right and then moved to the left side after endovascular embolization. This change was associated with a change in the vessel involved in the vascular malformation. In this observation, onset of CLH was at late adolescence whereas diagnosis of the AVM was made at 28 years of age following subarachnoid hemorrhage. At this time, the authors registered an impairment of blood flow in the vessel supplying the AVM by transcranial Doppler sonography. To explain the occurrence of $\mathrm{CLH}$, they hypothesized that there was an episodic sensitization of the whole cerebral vasculature due to the modification of blood flow. Attacks could be precipitated by environmental factors.

A further example of disorders of the vascular mechanism associated with CLH was given by Rowed [35]. The author described how arterial loops, the anterior inferior cerebellar artery or the internal auditory artery injured the nervus intermedius. Among the cases reported, he emphasized a case where symptoms of typical chronic refractory $\mathrm{CH}$ were relieved after surgical decompression of the nervus intermedius. A loop of the anterior inferior cerebellar artery linked to the seventh-eighth cranial nerve complex in the cerebellopontine angle, whereas the brainstem and trigeminal nerve were without abnormalities. In eight selected cases of severe chronic $\mathrm{CH}$, the authors conducted a prospective study searching for the presence of an arterial loop. This was found in six cases and treated by microvascular decompression of the nervus intermedius or the trigeminal main sensory root with limited postoperative complications. The result was considered to be successful as CLH symptoms were relieved. This study suggests that, in cases of chronic $\mathrm{CH}$, the presence of a vascular arterial loop should be investigated radiologically but does not indicate symptoms that might suggest their presence. Moreover, the number of cases was limited and no firm conclusion could be drawn.

Vertebral or carotid artery aneurysms are associated in rare cases with CLH [13, 36] although the temporal link between the two diseases is not clear. In West and Todman's case [36], CLH did not fulfill IHS criteria. On the contrary, Greve and Mai [13] observed a left aneurysm of the communicating artery which was responsible for subarachnoid hemorrhage. This aneurysm was disclosed after a long painfree period of typical CLH. The mechanism and the relationship between the diseases remain undetermined.

Arterial dissection is one of the less common causes of CLH. Cremer et al. [37] reported a case in which CLH occurred within two days of a fall responsible for right vertebral dissection. At first, oxygen, sumatriptan, methysergide and valproate worked well in curing or preventing the attacks. However, the usual periodicity and remission observed in $\mathrm{CH}$, as well as the constant efficacy of prophylactic drugs, were rapidly lacking. To link the diseases, the 
authors suggested a complex central mechanism for attacks implicating other structures such as those located in the cavernous sinus.

Carotid thrombendarterectomy has been described exceptionally in association with CLH. In one case, Bjorne et al. [38] reported a patient with a 22-year remission period from $\mathrm{CH}$ who experienced attacks on the day after carotid thrombendarterectomy. CLH was similar in all ways to that suffered two decades before and was located on the same side as the arterial vascular surgery. Damage to sympathetic fibers was identified by pharmacological and ophthalmologic tests. To explain this case, the authors postulated that CLH was possibly due to a sudden modification of carotid blood flow associated with transitory impairment of autonomic fibers of the face.

\section{CLH and other lesion sites}

This last section concerns several isolated cases reported in different areas of the brain with various etiologies. These cases are so exceptional that their relationship with CLH should be considered theoretical. A number of observations were selected with case heterogeneity in mind although, in this way, some cases may have been overlooked.

For tumor disease, De Angelis and Payne [39] reported a leptomeningeal lymphoma in which CLH accompanied the meningeal localization of the disease. If CLH was typical at onset, it was rapidly associated with signs of trigeminal mononeuropathy probably due to lesion of the trigeminal fibers by meningeal lymphoma. Other examples of tumoral diseases associated with CLH have been published but most of them did not fulfill IHS criteria. In most, clinical examination disclosed abnormalities requiring radiological tests $[15,17]$.

Midline intracranial calcification with extension into the third ventricle is another isolated example of a disorder showing CLH symptoms [40]. CH was typical in early years and diagnosed as chronic. In fact, several unusual features were observed and led to complementary tests. Among them, pain was not located in the orbital region, onset of disease was at the age of 60 years and $\mathrm{CH}$ seemed to be first chronic and then episodic. No mechanism was suggested to link the two diseases and the authors concluded the absence of a causal relationship. Nevertheless, calcification located close to the midline structure is suggestive of hypothalamic impairment potentially implicated in $\mathrm{CH}$ as previously discussed [4, 12].

Multiple sclerosis (MS) is a rare etiology of CLH because there are frequently neurological signs associated with pain that leads to suspicion of another neurological disease. However, Leandri et al. [41] reported a case in which CLH was the first sign of MS. The description adheres exactly to the IHS criteria and preventive therapy was also effective. A demyelination plaque was found by MRI in the pons at the trigeminal root entry zone. In this exceptional case, pain was not, as expected, trigeminal neuralgia pain but CLH pain. The authors hypothesized an interaction of the plaque with TVS causing altered activity of the trigeminal nerve.

Subclavian steal phenomenon was reported by Piovesan et al. [42] as a cause of CLH. Pain was also on the same side as the subclavian steal phenomenon resulting from an occluded subclavian artery. Blood flow was modified by thrombosis and demonstrated by angiography. Safenous vein bypass cured abnormal blood circulation and, 3 years later, no CLH was noted. The physiopathological mechanism suggested by the authors is based on hypoxia and increased blood flow in the internal carotid arteries [42]. This would provoke venous engorgement in the cavernous sinus and therefore CLH.

\section{Criteria for clinical practice}

As previously illustrated by the examples given, diagnosis of CLH remains difficult and frequently delayed. The criteria selected are disputable but provided a scope of like case observations. The criteria are based on the IHS classification and should identify most of the cases suspected as CLH [2].

Several signs or factors can suggest the occurrence of a second disease in cases of $\mathrm{CH}$. Obviously, one alone has no value. However, a number of associated factors in a single case increases the probability of CLH. Among them, authors seem to insist on sex and age at onset. As male preponderance is a well-known characteristic of $\mathrm{CH}$, occurrence in middle-age or older women should suggest CLH [3, 5]. Type and nature of pain is a factor of prime importance for authors in the suspicion of CLH. In fact, orbital burning sensation, moderate intensity of pain and absence of periodicity are frequently depicted in CLH $[31,39]$. These signs are almost always found retrospectively in published cases. With this in mind, description of facial pain should be very precise even with a standard questionnaire. The duration of attacks is another important point. Attacks exceeding 180 minutes rule out diagnosis of CLH [25]. Absence of autonomic symptoms $[16,18,27,33,34]$ or their presence outside attacks is sometimes found but in $\mathrm{CH}$, ptosis and miosis might persist inconstantly [1]. These signs appear, however, of less value than those of type of pain or duration of attacks. The last, but not the least important point is the quality of physical and clinical examinations. In fact, several cases prove that, even in the presence of IHS criteria for $\mathrm{CH}$, patient examination was abnormal [13, 25]. Clinical examination should be as complete as possible and not lim- 
ited to face and cranial nerve explorations. A general study is always required as some indirect signs of a lesion, such as galactorrhea, may be detected.
To conclude, the rarity of CLH should be emphasized. For this reason, radiological or electrophysiological examination is only exceptionally required in $\mathrm{CH}$.

\section{References}

1. Goadsby P (2001) Trigeminal autonomic cephalalgias (TACs). Acta Neurol Belg 101:10-19

2. - (1988) Classification and diagnosis criteria for headache disorders, cranial nevralgias and facial pain. Headache Classification Committee of the International Headache Society. Cephalalgia 8[Suppl 7]:1-96

3. Elkbom K (1999) Cluster headache: a clinical and pathophysiological overview. In: Olesen J, Goadsby PJ (eds) Cluster headache and related conditions. Oxford University, Oxford, pp 14-22

4. May A, Bahra A, Buchel C, Frakowiack RS, Goadsby PJ (1998) Hypothalamic activation in cluster headache. Lancet 352:275-278

5. Manzoni GC (1999) Cluster headache and lifestyle: remarks on a population of 374 male patients. Cephalalgia 19:88-94

6. Edvinsson L (1999) Neuropeptides in cluster headache, trigeminal neuralgia and chronic paroxysmal hemicrania. In: Olesen J, Goadsby PJ (eds) Cluster headache and related conditions. Oxford University, Oxford, pp 125-132

7. Moskowitz MA (1988) Cluster headache: evidence for a pathophysiologic focus in the superior pericarotid cavernous sinus plexus. Headache 28:584-586

8. Hardebo JE (1994) How cluster headache is explained as an intracavernous inflammatory process lesioning sympathetic fibers. Headache 34:25-31

9. Hardebo JE (1984) The involvement of trigeminal substance $P$ neurons in cluster headache. Headache 24:293-304

10. Sicuteri F (1985) Quasi phantom eye pain: an evolution of the substance $\mathrm{P}$ theory of cluster headache. Headache 25:447-451

11. Hannertz J, Ericson K, Bergstrand (1987) Orbital phlebography in patients with cluster headache. Cephalalgia 7:207-211
12. Sjaastad O (1988) Cluster headache: the possible significance of midline structures. Cephalalgia 8:229-236

13. Greve E, Mai J (1988) Clusterheadache like headaches: a symptomatic feature? A report of three patients with intracranial pathologic findings. Cephalalgia 8:75-82

14. Porta-Etassam J, Ramos-Carrasco A, Berbel-Garcia A, Martinez-Salio A, Benito-Leon J (2001) Clusterlike headache as first manifestation of prolactinoma. Headache 41:723-725

15. Kuritzky J (1984) Cluster headache like pain caused by an upper cervical meningioma. Cephalalgia 4:185-186

16. Masson C, Lehericy S, Guillaume B, Masson M (1995) Cluster-like headache in a patient with a trigeminal neurinoma. Headache 35:48-49

17. Taji J, Sas K, Szok D, Vörös E, Vecsei L (1996) Clusterlike headache as the first sign of brain metastases of lung cancer. Headache 36:259-260

18. Heidegger S, Mattfeldt T, Wikstroem M, Kern P, Kern W, Schreinber H (1997) Orbitosphenoidal aspergillus infection mimicking cluster headache. Cephalalgia 17:676-679

19. Evers S, Soros P, Brilla R, Gerding H, Husstedt IW (1997) Cluster headache after orbital exenteration. Cephalalgia 17:680-682

20. Rogado AZ, Graham JR (1979) Through a glass darkly. Headache 19:58-62

21. Sacquegna T, D'Allesandro R, Cortelli P, De Carolis P, Baltrati A (1982) Cluster headache after herpes zoster ophthalmicus. Arch Neurol 39:384

22. Takeshima T, Nishikawa S, Takahashi K (1988) Cluster headache like symptoms due to sinusitis: evidence for a neuronal pathogenesis of cluster headache syndrome. Headache 28:207-208

23. Romoli M, Cudia G (1988) Cluster headache due to an impacted superior wisdom tooth: case report. Headache 28:135-136
24. Salomom S (1986) Cluster headache and the nervus intermedius. Headache 26:3-8

25. Milos P, Havelius U, Hindfelt B (1996) Clusterlike headache in a patient with pituitary adenoma, with review of literature. Headache 36:184-188

26. Tfelt-Hansen P, Paulson OB, Krabbe AE (1982) Invasive adenoma of the pituitary gland and migrainous neuralgia. A rare coincidence or a causal relationship? Cephalalgia 2:25-28

27. Hannertz J (1989) A case of parasellar meningioma mimicking cluster headache. Cephalalgia 9:265-269

28. Lefevre JP, Simmat G, Bataille B, Salles M, Gil R, Boissonnot L, Rouades G (1984) Algies vasculaires de la face dues à un méningiome. Presse Méd 38:2323

29. Hall WA, Luciano MG, Doppman JL, Patronas NJ, Oldfield EH (1994) Pituitary magnetic resonance imaging in normal human volunteers: occult adenomas in the general population. Ann Intern Med 120:817-820

30. Burrow GN, Wortzman G, Rewcastle NB, Holgate RC, Kovacs K (1981) Microadenomas of the pituitary and abnormal sellar tomograms in unselected autopsy series. N Engl J Med 304:156-158

31. Zanchin G, Rossi P, Licanadro AM, Fortunato M, Maggioni F (1995) Cluster headache, a case of sphenoidal aspergilloma. Headache 35:494-497

32. Testa D, Frediani F, Bussone G (1988) Cluster headache like syndrome due to arteriovenous malformation. Headache 28:36-38

33. Hindfelt B, Olivecrona H (1991) Cerebral arteriovenous malformation and cluster like headache. Headache 31:514-517

34. Gawel MJ, Willinsky RA, Krajewski (1989) A reversal of cluster headache side following treatment of arteriovenous malformation. Headache 29:453-454 
35. Rowed DW (1990) Chronic cluster headache managed by nervus intermedius section. Headache 30:401-406

36. West P, Todman D (1991) Chronic cluster headache associated with vertebral artery aneurysm. Headache 31:210-212

37. Cremer PD, Halmagyi GM, Goadsby PJ (1995) Secondary cluster headache responsive to sumatriptan. J Neurol Neurosurg Psychiatry 59:633-634
38. Bjorne A, Hindfelt B, Havelius U (1994) Recurrence of cluster headache after carotid thrombendarterectomy. Headache 34:230-233

39. De Angelis LM, Payne R (1987) Lymphomatous meningitis presenting as atypical cluster headache. Pain 30:211-216

40. Narbone MC, D'Amico D, Di Maria F, Arena MG, Longo M (1991) Clusterlike headache and a median intracranial calcified lesion: case report. Headache 31:684-685
41. Leandri M, Cruccu G, Gottlieb A (1999) Cluster headache-like pain in multiple sclerosis. Cephalalgia 19:732-734

42. Piovesan EJ, Lange MC, Werneck LC, Kowacs PA, Engelhorn AL (2001)

Cluster-like headache. A case secondary to the subclavian steal phenomenon. Cephalalgia 21:850-851 